\title{
Perfil fonoaudiológico da comunidade do Dendê: perspectiva para ações futuras
}

\author{
Speech-language pathology profile of the Dendê community: \\ perspectives for future actions
}

\author{
Denise Klein Antunes ${ }^{1}$
}

\begin{abstract}
RESUMO
Objetivo: Traçar o perfil fonoaudiológico da comunidade do Dendê, caracterizando os aspectos de linguagem oral e escrita, voz, audição e motricidade orofacial, a partir de queixas referidas. Métodos: Estudo exploratório-descritivo, realizado a partir da aplicação de protocolo abordando aspectos gerais e fonoaudiológicos, com base nas informações contidas nos prontuários das 335 famílias assistidas pela Fonoaudiologia na comunidade do Dendê, totalizando 1704 sujeitos. Resultados: Grande parte das famílias do Dendê, $70 \%$, ganha até dois salários mínimos. Observou-se que 20,6\% da população infantil apresentou queixas de problemas de linguagem, sendo $21,2 \%$ delas, de dificuldade de aprendizagem escolar. Houve ocorrências do hábito de sucção de chupeta em 50,4\% das crianças, seguido da mamadeira em $42,2 \%$, com até dois anos de idade. Nas crianças entre dois e seis anos ainda observou-se uso elevado destes hábitos, sendo 31,2\% uso da mamadeira e 29,7\% sucção de chupeta. A sucção digital foi observada em $9,1 \%$ das crianças acima de seis anos. Houve um baixo percentual de queixas auditivas, assim como de voz, em apenas 2,2\% da população. Conclusão: Foi verificado que as alterações fonoaudiológicas mais prevalentes estavam relacionadas à presença de hábitos orais, seguidas por aspectos da linguagem oral e escrita. No entanto, um baixo nível de queixas de audição e de voz foi relatado. A realização de um diagnóstico situacional ajuda no redirecionamento das atividades postas em prática na comunidade, visando a população assistida. Isto é alcançado por meio de iniciativas educativas de prevenção e promoção de saúde, as quais devem conduzir a resultados melhores, mais rápidos e mais eficazes.
\end{abstract}

Descritores: Desenvolvimento da linguagem; Hábitos; Perfil de saúde; Patologia da fala e linguagem; Prevalência; Transtornos da linguagem; Voz; Audição; Sistema estomatognático

\section{INTRODUÇÃO}

Estabelecer a prevalência dos problemas da comunicação humana de uma determinada população é relevante para que se desenvolvam ações fonoaudiológicas mais efetivas.

Hoje em dia, poucos são os dados epidemiológicos sobre as alterações da comunicação humana na população no município de Fortaleza. É reduzido o número de instituições que têm o serviço de Fonoaudiologia gratuito para atender a demanda da cidade de Fortaleza, sendo o Núcleo de Atenção Médica Integrada (NAMI), clínica-escola da Universidade de Fortaleza (UNIFOR), referência no Nordeste. Este serviço presta assistência em todos os níveis de

Trabalho realizado na Universidade de Fortaleza - UNIFOR - Fortaleza (CE), Brasil, e no Núcleo de Atenção Médica Integrada - NAMI - Fortaleza (CE), Brasil.

(1) Mestre, Professora do Curso de Fonoaudiologia da Universidade de Fortaleza - UNIFOR - Fortaleza (CE), Brasil.

Endereço para correspondência: Denise Klein Antunes. R. Tibúrcio Cavalcante, 2150/2200, Dionísio Torres, Fortaleza (CE), Brasil, CEP: 60125-101.

E-mail: deniseklein@ secrel.com.br

Recebido em: 6/8/2009; Aceito em: 6/1/2010 atenção à saúde, tendo o setor de Fonoaudiologia realizado 34.547 atendimentos em 2007.

Durante o ano de 2006 e 2007, 58,09\% dos atendimentos para os distúrbios da comunicação ocorreram nas faixas etárias entre zero e dez anos. Destes, 36,99\% estão relacionados aos distúrbios da linguagem oral e escrita. A demanda tem sido absorvida pelo serviço através de triagens, orientações e grupo terapêutico familiar, oficinas, terapia individual e em grupo. Em 2007, a terapia em grupo atingiu percentual significativo, com $37,27 \%$ nos seus atendimentos, beneficiando a população que procura o serviço ${ }^{(1)}$. Assim, mudanças no modelo de atenção à saúde vêm ocorrendo em todas as áreas na lógica do Sistema Único de Saúde (SUS), enfatizando a função social da Saúde Pública ${ }^{(2,3)}$. Sob esse prisma, têm sido feitos investimentos em projetos que possibilitem uma aproximação entre instituições formadoras e o serviço, transformando as práticas pedagógicas e reorientando o modo de cuidar da saúde individual e coletiva.

Com base nas Diretrizes Curriculares Nacionais de 19/02/2002, os cursos de graduação em Fonoaudiologia no Brasil, vêm sendo reorientados, visando maior integração e inserção da profissão no Sistema de Saúde vigente e, atual- 
mente, mais diretamente através dos Núcleos de Apoio a Saúde da Família (NASF).

Inserir a Fonoaudiologia nas políticas de saúde e educação ${ }^{(4)}$ e valorizar práticas de saúde que possibilitem vivenciar realidades do SUS nos diversos cenários prepara o discente para a aproximação e o enfrentamento da realidade, com mudanças nos conhecimentos adquiridos, nas atitudes, habilidades, bem como para perceber melhor os fatores de risco aos quais à população está exposta ${ }^{(5)}$. Experiência de outra Universidade com discentes na saúde coletiva aponta para mudanças nos objetivos e metodologias pedagógicas utilizadas, contribuindo para o avanço na formação acadêmica e para população ${ }^{(6)}$.

Essa prática aproxima o fonoaudiólogo da população, procurando ouvi-la e melhor entende-la, facilitando transformações de acordo com as necessidades de saúde da comunidade $\operatorname{assistida~}^{(7)}$.

Partindo dessa visão, foi desenvolvido um trabalho interdisciplinar envolvendo as disciplinas da Saúde Coletiva dos cursos do Centro de Ciências da Saúde (CCS), da UNIFOR, juntamente com o NAMI. Essa proposta possibilitou a transformação das práticas destas disciplinas, realizadas na comunidade do Dendê, com aproximadamente 14.000 habitantes, situada próximo à UNIFOR.

Deste modo, realizar o diagnóstico situacional in loco é o primeiro passo para o planejamento de ações educativas em saúde mais efetivas. A partir do perfil identificado, faz-se então necessário definir prioridades com resolutividade compatível com a condição de vida, visando autonomia da comunidade em relação a uma comunicação saudável ${ }^{(8)}$.

Portanto, o objetivo desta pesquisa foi traçar o perfil fonoaudiológico da comunidade do Dendê, caracterizando os aspectos de linguagem oral e escrita, motricidade orofacial, audição e voz, a partir das queixas referidas.

\section{MÉTODOS}

Trata-se de um estudo exploratório-descritivo, com abordagem quantitativa, realizado no período de junho de 2006 a fevereiro de 2007, aprovado pelo Comitê de Ética em Pesquisa da instituição com o parecer $n^{\circ}$ 228/2006, segundo Resolução $n^{\circ}$ 196/96 (CNS).

A comunidade do Dendê está localizada próxima à instituição é formada por aproximadamente 14.000 sujeitos de baixa renda, com famílias morando em espaços de invasão como a área Chico Mendes e a Baixada do Aratu, sendo esta, considerada como Área de Preservação Permanente (APP), que compreende uma área de manguezal, às margens do Rio Cocó $^{(9)}$. Essa comunidade se beneficia dos serviços do NAMI. Este, desde agosto de 2006, desenvolve suas atividades práticas de Saúde Coletiva do Centro de Ciências da Saúde (CCS) de acordo com a lógica do Programa de Saúde da Família (PSF), coberta por quatro equipes em parceria com um Centro de Saúde da Família da Secretaria Executiva Regional VI, da prefeitura. O trabalho domiciliar com as famílias desta comunidade foi iniciado, pelo curso de Fonoaudiologia, em abril de 2002.

Para a realização da pesquisa foram utilizados todos os prontuários das 335 famílias, assistidas pela Fonoaudiolo- gia, com o total de 1704 sujeitos. Considerou-se o registro dos dados da primeira visita realizada à família, indicada no prontuário. Foram excluídos da pesquisa os prontuários com dados incompletos, que dificultaram a análise dos resultados.

Aplicou-se um protocolo pré-estabelecido com base nos prontuários das famílias, referentes a aspectos gerais e fonoaudiológicos, considerando as seguintes variáveis: renda, idade, escolaridade, queixas de alterações de linguagem oral e escrita, hábitos orais, audição e voz.

Cabe referir que os dados registrados nos prontuários foram coletados durante visitas domiciliares realizadas pelos próprios alunos, com supervisão de professores e monitores na prática da disciplina de Fonoaudiologia na Saúde Coletiva, do $6^{\circ}$ semestre do curso de Fonoaudiologia, no período de fevereiro de 2002 a novembro de 2005. Realizaram diagnóstico situacional, planejamento, execução e monitoramento das intervenções desenvolvidas. Para a pesquisa, contemplou-se apenas a exploração do diagnóstico situacional com a detecção das queixas relacionadas aos aspectos fonoaudiológicos, de acordo com a realidade de cada família visitada.

Para o processamento e análise dos dados foram utilizados os programas Epi Info versão 6.04 e SPSS.

\section{RESULTADOS}

Quanto à renda familiar, grande parte das famílias do Dendê, 223 (70\%), ganha até dois salários mínimos, sendo 65 $(20,4 \%)$ sem renda ou ganhando menos de um salário mínimo.

Em relação à faixa etária, a de maior prevalência foi de indivíduos entre 30 a 60 anos, 474 (27,8\%). Apesar de a concentração ser na fase adulta, observa-se na Tabela 1 que quase um quarto $412(24,1 \%)$ da população pesquisada é de crianças.

Tabela 1. Caracterização da amostra dos sujeitos pertencentes à comunidade do Dendê, em Fortaleza (CE), de acordo com a faixa etária $(\mathrm{N}=1704)$

\begin{tabular}{lcc}
\hline Idade & $\mathrm{N}$ & $\%$ \\
\hline $0-\mid 2$ & 109 & 6,4 \\
$2-\mid 5$ & 128 & 7,5 \\
$5-10$ & 175 & 10,3 \\
$10 \mid-20$ & 346 & 20,3 \\
$20 \mid-30$ & 348 & 20,5 \\
$30 \mid-60$ & 474 & 27,8 \\
$60 \mid-\infty$ & 108 & 6,3 \\
Não responderam & 16 & 0,9 \\
\hline
\end{tabular}

Conforme apresentado na Tabela 2, cerca de $90 \%$ da população foi alfabetizada. Foi também demonstrada uma equivalência nesta população entre as faixas etárias das crianças e de adultos jovens. No entanto, esse índice apresentou uma redução gradativa com o aumento da idade, visto que à proporção que a faixa etária ia aumentando, o percentual de alfabetizados ia diminuindo. Esse dado é observado de 30 a 60 anos, $376(79,3 \%)$ e com mais de 60 anos, em que 53 (49,5\%) foram alfabetizados.

Em relação à ocorrência de problemas de linguagem e de dificuldade de aprendizagem, verifica-se na Tabela 3 que 
Tabela 2. Caracterização de uma amostra de sujeitos alfabetizados e não alfabetizados, por faixa etária, pertencentes à comunidade do Dendê $(\mathrm{N}=1435)$

\begin{tabular}{lccc}
\hline \multirow{2}{*}{ Idade (anos) } & \multicolumn{2}{c}{ Alfabetizados } & Total \\
\hline$>6$ e $<10$ & Sim $(\%)$ & Não $(\%)$ & \\
$>10$ e $<20$ & $323(93,8,5)$ & $19(11,5)$ & 165 \\
$>20$ e $<30$ & $309(90,1)$ & $34(9,9)$ & 346 \\
$>30$ e $<60$ & $376(79,3)$ & $98(20,7)$ & 474 \\
$>60$ & $53(49,5)$ & $54(50,5)$ & 107 \\
\hline
\end{tabular}

$20,6 \%$ do total de 126 crianças em idade pré-escolar e que $17,6 \%$ do total de 165 crianças, pertencentes à faixa de seis a dez anos, apresentaram queixas de problemas de linguagem. Esse percentual foi reduzido, significativamente, na adolescência, chegando a 1,7\%. As queixas de dificuldade de aprendizagem escolar tornaram-se um pouco mais elevadas quando comparadas às queixas de linguagem, uma vez que 35 crianças $(21,2 \%)$ em idade escolar e 29 adolescentes $(8,3 \%)$ apresentaram problemas referentes à aprendizagem.

Tabela 3. Relação entre queixas de problemas de linguagem e de dificuldade de aprendizagem, por faixa etária, com sujeitos de $\geq 6$ a $<20$ anos, pertencentes à comunidade do Dendê $(\mathrm{N}=511)$

\begin{tabular}{lcccc}
\hline Queixa & \multicolumn{5}{c}{ Idade (anos) } \\
& $N$ e $<6$ & $\geq 6$ e $<10$ & $\geq 10$ e $<20$ & Valor de $p$ \\
& $N(126)$ & $N(165)$ & $N(346)$ & \\
\hline Problemas de linguagem & & & \\
\hline Sim & $26(20,6 \%)$ & $29(17,6 \%)$ & $6(1,7 \%)$ & 0,000 \\
Não & $100(79,4 \%)$ & $136(82,4 \%)$ & $340(98,3 \%)$ & \\
\hline
\end{tabular}

Dificuldade de aprendizagem

\begin{tabular}{llccc}
\hline Sim & - & $35(21,2 \%)$ & $29(8,3 \%)$ & 0,000 \\
Não & - & $130(78,9 \%)$ & $317(91,7 \%)$ & \\
\hline
\end{tabular}

Teste do Qui-quadrado $(\mathrm{p} \leq 0,05)$

Em relação aos hábitos orais observa-se maior ocorrência do hábito de chupeta com 55 crianças $(50,4 \%)$, seguido da mamadeira com $46(42,2 \%)$ nas crianças com até dois anos de idade. Da mesma forma, os resultados apontaram, ainda, a ocorrencia elevada destes hábitos entre dois e seis anos, sendo $40(31,2 \%)$ mamadeira e $38(29,7 \%)$ chupeta. A análise mostrou que o uso da chupeta foi o mais frequiente entre a população infantil, mesmo nos grupos com idade mais avançada. Já em relação à sucção digital observou-se uma inversão nos dados, sendo, este hábito mais frequente na idade escolar, entre seis e dez anos, em 9,1\%. Das 148 crianças até dez anos que apresentaram hábitos, 66 (44,5\%) e $23(15,4 \%)$ também apresentaram dois e três hábitos associados, respectivamente.

Na Tabela 4 observa-se a relação entre a dificuldade na introdução de consistências alimentares (DICA) com idade e presença de hábitos orais associados, dentre estes o uso da mamadeira e chupeta. Do total de crianças, $28(25,7 \%)$ de 109 apresentaram DICA na faixa etária até dois anos, reduzindo essa dificuldade, gradativamente, com a idade. Das 101 crianças que usavam mamadeira, 30 (29,7\%) apresentaram dificuldade na DICA, bem como das 114 que usavam chupeta, $30(26,3 \%)$.

Tabela 4. Relação entre a dificuldade de introdução de consistências alimentares com a idade, uso da mamadeira e chupeta, em crianças menores que dez anos pertencentes à comunidade do Dendê

\begin{tabular}{lcccc}
\hline Variável & $\mathrm{N}$ & \multicolumn{3}{c}{ DICA } \\
& & Sim (\%) & Não (\%) & Valor de $\mathrm{p}$ \\
\hline Idade & & & & \\
$0-\mid 2$ & 109 & $28(25,7)$ & $81(85,7)$ & \\
$2-\mid 6$ & 128 & $15(11,7)$ & $113(88,3)$ & 0,000 \\
$6-\mid 10$ & 175 & $2(1,1)$ & $173(98,9)$ & \\
Mamadeira & & & & \\
$\quad \begin{array}{l}\text { Presente } \\
\text { Ausente }\end{array}$ & 101 & $30(29,7)$ & $71(70,3)$ & 0,000 \\
Chupeta & 301 & $15(4,8)$ & $296(95,2)$ & \\
$\begin{array}{l}\text { Presente } \\
\text { Ausente }\end{array}$ & 114 & $30(26,3)$ & $84(73,7)$ & 0,000 \\
\hline
\end{tabular}

Teste do Qui-quadrado $(\mathrm{p} \leq 0,05)$

Legenda: $\mathrm{DICA}$ = dificuldade de introdução de consistências alimentares

A otite foi à queixa auditiva mais referida pela população com menos de 60 anos, sendo a faixa etária de zero a dez anos a mais acometida $(23-17,2 \%)$, seguida pela faixa etária de dez a 20 anos $(7-3,8 \%)$, e de 20 a 60 anos $(22-2,7 \%)$. Para a população acima de 60 anos a perda auditiva, a vertigem e o zumbido foram as queixas de maior prevalência, com 12 $(11,1 \%)$, nove $(8,3 \%)$ e oito $(7,4 \%)$ ocorrências, respectivamente, mencionadas na Tabela 5.

Tabela 5. Distribuição por idade das queixas auditivas, dos sujeitos pertencentes à comunidade do Dendê $(\mathrm{N}=1704)$

\begin{tabular}{|c|c|c|c|c|c|c|c|c|c|}
\hline \multirow{3}{*}{ Idade } & \multirow{3}{*}{$\mathrm{N}$} & \multicolumn{8}{|c|}{ Queixas auditivas } \\
\hline & & \multicolumn{2}{|c|}{ Otite } & \multicolumn{2}{|c|}{$\begin{array}{c}\text { Perda } \\
\text { auditiva }\end{array}$} & \multicolumn{2}{|c|}{ Zumbido } & \multicolumn{2}{|c|}{ Vertigem } \\
\hline & & Sim & $\%$ & Sim & $\%$ & Sim & $\%$ & Sim & $\%$ \\
\hline $0-10$ & 412 & 23 & 17,2 & 13 & 9,2 & 7 & 4,9 & 5 & 3,4 \\
\hline $10 \mid-20$ & 346 & 7 & 3,8 & 5 & 2,7 & 6 & 3,7 & 4 & 2,4 \\
\hline $20 \mid-60$ & 822 & 22 & 2,7 & 20 & 2,4 & 20 & 2,4 & 14 & 1,8 \\
\hline $60 \mid-\infty$ & 108 & 5 & 4,6 & 12 & 11,1 & 8 & 7,4 & 9 & 8,3 \\
\hline Não responderam & 16 & - & - & - & - & - & - & - & - \\
\hline Total & 1704 & 57 & 28,3 & 50 & 25,4 & 36 & 18,4 & 31 & 15,9 \\
\hline
\end{tabular}

Assim como as queixas auditivas apontaram um índice reduzido, o mesmo ocorreu com as queixas vocais, referidas por apenas $2,2 \%$ do total da população.

\section{DISCUSSÃO}

No presente estudo as famílias pesquisadas, em sua maioria apresentavam renda familiar de até dois salários mínimos. Em estudo anterior ${ }^{(9)}$ a maioria das famílias residentes na comunidade no ano de 1999, ganhava mais de um salário mínimo. Outro estudo que mostra um recorte desta comunidade ${ }^{(10)}$ revela 
que 158 famílias $(49,5 \%)$ ganham entre um e dois salários mínimos sendo $65(20,4 \%)$ sem renda ou ganhando menos de um salário mínimo. Assim, verificou-se semelhança com o observado nos dias atuais em que a população em estudo, ainda permanece em condições econômicas desfavoráveis.

Neste estudo anterior da comunidade do Dendê( ${ }^{(9)}$, havia crianças menores que cinco anos em quase metade das famílias, evidenciando nos achados desta pesquisa uma diminuição para $412(24,1 \%)$ crianças.

Por ter quase um quarto da população representada por crianças, há uma necessidade de maior atenção às mesmas. Sabe-se que o desenvolvimento da linguagem é um fator preponderante na comunicação humana, podendo ser influenciado pelo baixo nível socioeconômico da família e escolaridade dos pais, pelo contexto familiar, conflitos e a falta de estimulação ${ }^{(5)}$.

Foi verificado que o percentual das crianças que apresentaram queixas de problemas de linguagem com idade entre seis e dez anos é muito próximo da proporção de crianças com queixas de problemas de aprendizagem escolar. Cerca de $12 \%$ das crianças no início da escolaridade apresenta alteração de linguagem ${ }^{(11)}$. Nessa fase, observou-se que praticamente todas as crianças estavam alfabetizadas, não significando dizer, no entanto, que existe um bom nível escolar, visto que o instrumento aplicado na coleta de dados não apresentou variável para classificar o desempenho escolar dos integrantes das famílias.

Em pesquisa realizada, apenas metade das pessoas entre seis e quinze anos frequientava escola ${ }^{(10)}$. Segundo dados do IBGE $^{(12)}$, a população dessa comunidade permanece na escola de cinco a oito anos sendo um tempo reduzido considerando que, nesse período, os jovens têm concluído pouco mais que o ensino fundamental. Corroborando o estudo, crianças pertencentes à população carente, além de inseridas tardiamente, têm um tempo de permanência insuficiente no contexto escolar, retardando o início e a qualidade do processo educativo, o que as deixam em desvantagem se comparadas com as demais crianças das escolas particulares. Estas costumam não só entrar precocemente, mas permanecer o tempo adequado na realidade escolar ${ }^{(13)}$.

Os dados também revelaram um percentual importante de queixas de dificuldade de aprendizagem escolar, representado por 64 (29,5\%), um terço da população estudada, podendo estar vinculado a fatores como os de razão sócio-econômica, bem como às relações familiares ${ }^{(5,14,15)}$.

Outro estudo sobre dificuldades de aprendizagem em escolares de diferentes níveis socioeconômicos, afirma que os mais carentes passam por várias privações e desestruturações familiares, em que os pais não estão acostumados a valorizar o espaço escolar e a participar ativamente da construção de conhecimento dos filhos, repercutindo, no desempenho educativo $^{(16)}$.

Associado aos fatores como renda e nível educacional familiar há que ser considerada como influência nas dificuldades das crianças, a possibilidade de entrada tardia na escola, metodologia de ensino e o nível de escolaridade do professor, que podem, também, contribuir para o aumento das queixas de dificuldades de aprendizagem escolar que, em maior parte, costumam ser vinculados somente ao aluno.

Outro fator importante que pode interferir na comunicação humana são os hábitos orais. Na amostra, 303 (73,6\%) são crianças na faixa etária entre dois e dez anos de idade. Destas, $137(33,2 \%)$ apresentaram algum hábito oral. Nessa fase é esperado que os hábitos já tenham sido removidos, mesmo que de forma gradativa com o decorrer da idade. Foi verificada, na pesquisa, uma redução mais significativa apenas a partir dos seis anos. Esse é um período considerado de risco para o desencadeamento de alterações seguido de possíveis distúrbios orofaciais, conforme aumento da idade como: mau posicionamento dentário, desvios no crescimento maxilar, distúrbios da deglutição e fonação ${ }^{(17,18)}$.

Desta forma, por se tratar de uma comunidade de baixa renda é relevante investir na perspectiva da promoção da saúde, com atuação preventiva e educativa facilitando o acesso às informações ${ }^{(19)}$. Essa visão possibilita reduzir os fatores de risco aos problemas orais e de comunicação, devendo o hábito oral ser retirado o quanto antes minimizando, consequentemente, o investimento dos cuidados assistenciais, em nível secundário.

Há estudos que apontam uma predisposição das crianças prolongarem o hábito como mecanismo para compensar a ausência dos pais, podendo interferir diretamente na alimentação ${ }^{(17,18)}$. Das crianças com até dois anos $28(25,7 \%)$, ou seja, um quarto apresentou dificuldade na transição alimentar e, 60 $(38,5 \%)$ ainda mantinha algum hábito oral. Tal constatação pode, também, estar relacionada com a dificuldade de acesso a alimentos nutritivos e essenciais para o crescimento da criança. Os dados obtidos na pesquisa não permitiram relacionar a amamentação com a consistência alimentar. No entanto, evidenciou-se relação da associação da consistência alimentar com a permanência dos hábitos. Trinta $(29,7 \%)$ crianças que faziam uso de mamadeira e $30(26,3 \%)$ usavam chupeta, apresentaram dificuldade na transição alimentar.

Em relação às queixas auditivas mencionadas, a otite foi a de maior prevalência na faixa etária de zero a dez anos 23 $(17,2 \%)$. As otites médias representam um terço do atendimento pediátrico de crianças de um a cinco anos. Autores relatam que a otite pode vir acompanhada por perdas auditivas condutivas, episódicas e variáveis, variando de grau leve a moderado, portanto, considerado um problema de saúde pública, uma vez que a doença tem impacto significativo na criança, na família e no sistema de assistência médica ${ }^{(20,21)}$.

Foi observada, também, a queixa de otite na faixa etária acima de 60 anos em 4,6\% dos indivíduos, estando relacionada às condições de vida da população como habitação, higiene (uso de cotonetes, palitos, grampos, tampas de caneta, e outros objetos para manipulação da orelha), nutrição inadequada, bem como a precária atenção a saúde e a carência de informações $^{(22,23)}$.

Para a faixa etária acima de 60 anos, a perda auditiva foi a queixa mais relatada $12(11,1 \%)$. Estudos revelam que, com o aumento da idade, a perda auditiva pode aparecer, causando problemas na comunicação, levando a um isolamento e frustração a estes indivíduos ${ }^{(22)}$, podendo haver um aparecimento maior de múltiplos sintomas otoneurológicos associados, tais como, vertigem, perda auditiva, zumbido, entre outros, devido à perda da função vestibular ${ }^{(24,25)}$.

Foram encontradas, na população pesquisada acima de 60 anos, oito pessoas $(7,4 \%)$ com queixa de zumbido. Doze por 
cento dos indivíduos entre 65 a 74 anos são acometidos pelo zumbido ${ }^{(26)}$. Chega a atingir $15 \%$ da população geral, podendo $15 \%$ a $25 \%$ deste grupo, apresentar interferência importante na sua qualidade de vida ${ }^{(27,28)}$.

A pesquisa revela baixo percentual em todas as queixas auditivas referidas pela população, bem como queixas de voz, referidas por apenas 2,2\% do total da população. Contudo, a partir dos resultados encontrados, algumas considerações podem ser levantadas. É importante apontar o fato de a população estudada pertencer a uma classe sócio-econômica desfavorecida, em que se esperava encontrar um maior número de problemas auditivos, discordando de outros autores que afirmam que tais condições sócio-econômicas cooperam para fazer da população de baixa renda, uma população de risco para problemas auditivos e de saúde geral ${ }^{(29,30)}$.

Vale ressaltar, que alguns fatores contribuíram de forma direta para a limitação deste estudo, dificultando as estratégias de coleta e análise dos resultados: a coleta foi realizada em um período anterior ao início da pesquisa; o prontuário foi planejado para ser utilizado na disciplina de Fonoaudiologia na Saúde Coletiva, desde o início das atividades; os dados constantes nos prontuários foram construídos por alunos que cursaram a disciplina ao longo desse período, inviabilizando a uniformidade de registros, porém não invalidando estes dados.

\section{CONCLUSÃO}

Foi verificado que as mudanças fonoaudiológicas mais prevalentes estavam relacionadas à presença de hábitos orais, sendo mais freqüentes os de sucção de chupeta, seguido da mamadeira.

A faixa etária em que houve a maior presença de hábitos orais foi entre dois e seis anos, idade considerada de risco para desenvolvimento de alterações ao sistema estomatognático.

Há a presença de mais de um hábito em todas as idades, seguidas por aspectos da linguagem oral e escrita em quase um terço da população jovem. A relação das queixas de problemas de linguagem com as de dificuldade de aprendizagem escolar é praticamente a mesma, tanto na faixa etária de crianças e adolescentes.

No entanto, um baixo nível de queixas de audição e de voz foi relatado. A realização de um diagnóstico situacional ajuda no redirecionamento das atividades na prática com a comunidade, visando a sua população. Isto é alcançado através de iniciativas educativas de prevenção e promoção de saúde, as quais devem conduzir a resultados melhores e mais rápidos.

Conhecer a realidade social, o ambiente familiar e as relações das famílias assistidas, possibilita a maior compreensão de suas reais necessidades. Diante dos resultados, ressalta-se a importância de reestruturar o protocolo existente e a abordagem diante das famílias na coleta dos dados, para possibilitar informações mais precisas, estabelecendo melhoria nos determinantes e condicionantes da saúde fonoaudiológica.

\section{AGRADECIMENTOS}

Agradeço aos fonoaudiólogos Aline Torres de Menezes, Ana Clara Rocha de Souza, Emília Estela Freire Santiago, Eucária Carneiro Farias de França, Flávia Cristina Barbosa Carneiro, Laydiany Cavalcante Nogueira Lima, Luiziana Aires de Queiroz Farias, Wilker Sávio Gomes de Carvalho, pela contribuição através dos trabalhos de conclusão de curso, como subprojetos frutos desta pesquisa.

\begin{abstract}
Purpose: To trace the Speech-Language Pathology profile of the Dendê community, characterizing aspects of oral and written language, voice, hearing and orofacial myology, based on referred complaints. Methods: Exploratory-descriptive study, carried out with the application of a protocol that addressed general and Speech-Language Pathology aspects, based on information available on the records of 335 families assisted by Speech-Language Pathology at the Dendê community, totalizing 1704 subjects. Results: Great part of the families at Dendê (70\%) earns up to two minimum wages. It was observed that $20.6 \%$ of the children had language complaints, and $21.2 \%$ of these complaints regarded learning difficulties. Pacifier sucking habits were observed in $50.4 \%$ of the children, and $42.2 \%$ of the children up to two years old used nursing bottles. Children between two and six years old also presented high occurrence of these habits: $31.2 \%$ used nursing bottles and $29.7 \%$ used pacifiers. Thumb sucking was observed among $9.1 \%$ of the children older than six years old. There was a low percentage of hearing and voice complaints, reported by $2.2 \%$ of the population. Conclusion: It was verified that most prevalent Speech-Language Pathology alterations were related to the presence of oral habits, followed by oral and written language aspects. Nevertheless, a low level of hearing and voice complaints were reported. A situational diagnosis helps redirecting the activities practiced in the community, aiming its population. This is achieved through educational initiatives of health prevention and promotion, which should lead to better, quicker and more efficient results.
\end{abstract}

Keywords: Language development; Habits; Health profile; Speech-language pathology; Prevalence; Language disorders; Voice; Hearing; Stomatognathic system 


\section{REFERÊNCIAS}

1. Silva MEML, Regis ACF, Brasil CCP. O desafio do Nami-Unifor frente à necessidade da inserção do fonoaudiólogo na rede municipal de saúde de Fortaleza [trabalho de conclusão de curso]. Fortaleza: Universidade de Fortaleza; 2008.

2. Maia SM. Implicações sociais do trabalho fonoaudiológico na atenção primária à saúde. In: Befi D. Fonoaudiologia na atenção primária à saúde. São Paulo: Lovise; 1997.

3. Freire RM. Fonoaudiologia em saúde pública. Rev Saúde Pública. 1992;26(3):179-84.

4. Goulart BNG. A Fonoaudiologia e suas inserções no sistema único de saúde: análise prospectiva. Rev Fonoaudiol Brasil. 2003;4(3):29-34.

5. Antunes DK. A construção cultural da "evolução" da linguagem infantil: um repensar da prática fonoaudiológica [dissertação]. Fortaleza: Universidade de Fortaleza; 2001.

6. Marin CR, Chun RYS, Silva RC, Fedosse E, Leonelli BS. Promoção da saúde em fonoaudiologia: ações coletivas em equipamentos de saúde e educação. Rev Soc Bras Fonoaudiol. 2003;8(1):35-41.

7. Penteado RZ, Servilha EAM. Fonoaudiologia em saúde pública/coletiva: compreendendo prevenção e o paradigma da promoção da saúde. Distúrb Comun. 2004;16(1):107-16.

8. Mendes D, Vianna RD. Educação em saúde: tendência atual. In: Vieira RM, Vieira MM, Ávila CRB, Pereira LD. Fonoaudiologia na saúde pública. Carapicuíba: Pró-fono; 1995.

9. Pordeus AMJ, Farias FSAB, Oliveira ER, Moreira TP. Comunidade do Dendê: um diagnóstico de suas famílias. Rev Cent Ciênc Saúde (St. Maria). 1999;12(11):9-17.

10. Diniz RCM, Fraga MNO. O contexto social e epidemiológico dos moradores assentados em área de manguezal. Rev Bras Promoção Saúde. 2005;8(2):98-104.

11. Tomblin JB, Records NL, Buckwalter P, Zhang X, Smith E, O'Brien M. Prevalence of specific language impairment in kindergarten children. J Speech Lang Hear Res. 1997;40(6):1245-60.

12. Brasil. Instituto Brasileiro de Geografia e Estatística. Educação: resultados da Amostra. Censo Demográfico. IBGE [Internet]. 2000 [citado 2007 Ago 04];. Disponível em: http://www.ibge.gov.br/home/ estatistica/populacao/censo2000/educacao/tabela_regioes.shtm

13. Santos LLCP, Vieira LMF. "Agora seu filho entra mais cedo na escola": a criança de seis anos no ensino fundamental de nove anos em Minas Gerais. Revista Educ Soc. 2006;27(96):775-96.

14. Szymanski H. Práticas educativas familiares: a família como foco de atenção psicoeducacional. Estud Psicol (Campinas). 2004;21(2):5-16.

15. Rodrigues MCC, Mello RR, Fonseca SC. Dificuldade de aprendizagem em escolares de muito baixo peso ao nascer. J Pediatr. 2006;82(1):6-14.

16. Suehiro ACB. Dificuldade de aprendizagem da escrita num grupo de crianças do ensino fundamental. Psic Rev Psicol Vetor Ed. 2006;7(1):59-68.
17. Tomita NE, Sheihan A, Bijella VT, Franco LJ. Relação entre determinantes socioeconômicos e hábitos bucais de risco para másoclusões em pré-escolares. Pesqui Odontol Bras. 2000;14(2):169-75.

18. Cavassani VGS, Ribeiro SG, Nemr NK, Greco AM, Köhle J, Lehn CN. Hábitos orais de sucção: estudo piloto em população de baixa renda. Rev Bras Otorrinolaringol. 2003;69(1):106-10.

19. Araújo JJ, Pereira AFF, Bassi AKZ, Caldana ML, Bastos JRM. Promoção de saúde materno-infantil In: Bastos JRM, Peres SHCS, Caldana ML. Educação em saúde com enfoque em odontologia e em Fonoaudiologia. São Paulo: Santos; 2007. p. 35-42.

20. Saes SO, Goldberg TBL, Montovani JC. Secreção na orelha média em lactentes: ocorrência, recorrência e aspectos relacionados. J Pediatr (Rio J.). 2005;18(2):133-8.

21. Castanho RM, Braga TMS. Indicadores do desempenho escolar em crianças com otite média crônica. Temas Desenvolv. 2006;15(85/86):3844.

22. Figueiredo RR, Fabri ML, Machado WS. Otite externa difusa aguda: um estudo prospectivo no verão do Rio de Janeiro. Rev Bras Otorrinolaringol. 2004;70(2):226-31.

23. Morimoto WT, Medina NH, Guardado AN, Lima, MHA, Pinto AMM, Magnusson IM. Avaliação visual e auditiva em adultos e idosos na atenção básica. Bol Epidemiol Paul. 2006;30(3):2-8.

24. Amaral LCG, Sena APRC. Perfil audiológico dos pacientes da terceira idade atendidos no Núcleo de Atenção Médica Integrada da Universidade de Fortaleza. Fono Atual. 2004;7(27):58-64.

25. Ganança FF, Gazzola JM, Aratani MC, Perracini MR, Ganança MM. Circunstâncias e conseqüências de quedas em idosos com vestibulopatia crônica. Rev Bras Otorrinolaringol. 2006;72(3):388-93.

26. National Institute on Deafness and Other Communication Disorders. Statistics about hearing disorders, ear infections, and deafness. NIDCD [Internet]. [cited 2007 Nov 02]; Available from: <http://www.nidcd.nih. gov/health/statistics/hearing.asp

27. Ferrari GMS, Sanchez TG, Pedalini MEB. A eficácia do molde aberto para o controle do zumbido. Rev Bras Otorrinolaringol. 2007;73(3):3707.

28. Fowler EP. Head noises in normal and in normal and disordered ears: significance, measurement, differentiation and treatment. Arch Otolaryngol. 1944;39:498.

29. Lichtig I. Audição: Abordagens Atuais. Carapicuíba: Pró- Fono; 1997.

30. Sampaio FMO. Estudo da perda auditiva em crianças de zero a 14 anos atendidas em um serviço de referência [dissertação]. Fortaleza: Universidade Federal do Ceará; 2001. 